\title{
Epidemiology of prehospital emergency medical service use in Korean children
}

\author{
Se Uk Lee ${ }^{1}$, Dongbum Suh ${ }^{2}$, Hahn Bom Kim ${ }^{3}$, Jin Hee Jung ${ }^{4}$, \\ Ki Jeong Hong ${ }^{4}$, Jin Hee Lee ${ }^{2}$, Hye Young Jang ${ }^{5}$, Hyun Noh ${ }^{6}$, \\ Jae Yun Jung ${ }^{7}$, Do Kyun Kim², Young Ho Kwak ${ }^{2}$
}

\begin{abstract}
Department of Emergency Medicine, ${ }^{1}$ Samsung Medical Center, Sungkyunkwan University School of Medicine, Seoul, ${ }^{2}$ Seoul National University Hospital, Seoul, ${ }^{3}$ Seoul Medical Center, Seoul, ${ }^{4}$ Seoul Metropolitan Government Seoul National University Boramae Medical Center, Seoul, ${ }^{5}$ Soonchunhyang University Seoul Hospital, Seoul, ${ }^{6}$ Soonchunhyang University Bucheon Hospital, Bucheon, ${ }^{7}$ Seoul National University Bundang Hospital, Seongnam, Korea
\end{abstract}

Objective The aim of this study was to elucidate the epidemiology of pediatric patients transported by the National 119 Rescue Services in Korea.

Methods We enrolled all pediatric patients ( $<16$ years old) who used the National 119 Rescue Services in Korea between January 2006 and December 2008, and analyzed the 119 ambulance patient care record databases.

Results The total number of the cases was 238,644 for 3 years. The median age was 6 years old and 59.0\% were male, and the 2- to 5-year-old group was the largest (31.0\%). The peak transport times were in the afternoon (from 12:00 p.m. to 17:59 p.m., 36.3\%), on Saturday and Sunday (15.9\% and 15.7\%), and in summer (June to August, $27.3 \%$ ). The ratio of disease versus injury as the cause of the transports was $42.3 \%$ vs. 57.7\%. Among the 16 metropolitan cities and provinces, Gyeonggi (25.7\%), Seoul (17.6\%), and Incheon (7.0\%) account for almost half of the all transported children. Regarding the annual transport rates per 100,000 children standardized by age, and gender to the Korean child population, Jeju was the largest $(1,650.2)$ followed by Gangwon (1,201.3), and Jeonnam $(1,178.1)$.

Conclusion This report presents comprehensive epidemiologic data of pediatric patients transported by 119 rescue services in Korea.

Keywords Child; Emergency medical services; Epidemiology

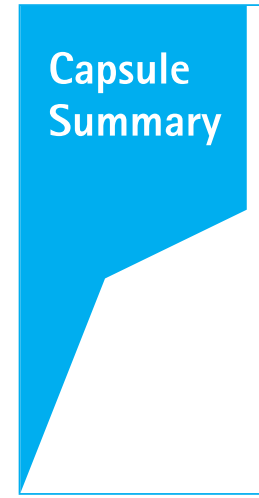

What is already known

Understanding the features of the emergency medical service system is essential to achieve improvements in the emergency medical service system.

\section{What is new in the current study}

The demographic and clinical characteristics of the transported pediatric patients in Korea are reviewed, and a comparison of pediatric prehospital transports among metropolitan cities and provinces in Korea is demonstrated.
Received: 21 January 2017

Revised: 31 March 2017

Accepted: 6 April 2017

Correspondence to: Young Ho Kwak Department of Emergency Medicine, Seoul National University Hospital, 101 Daehak-ro, Jongno-gu, Seoul 03080, Korea

E-mail:yhkwak@snuh.org

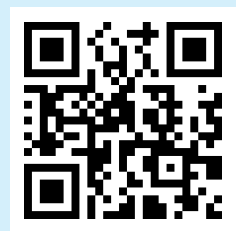

How to cite this article:

Lee SU, Suh D, Kim HB, Jung JH, Hong KJ, Lee JH, Jang HY, Noh H, Jung JY, Kim DK, Kwak YH. Epidemiology of prehospital emergency medical service use in Korean children. Clin Exp Emerg Med 2017;4(2):102108.

This is an Open Access article distributed under the terms of the Creative Commons Attribution Non-Commercial License (http:// creativecommons.org/licenses/by-nc/4.0/). 


\section{INTRODUCTION}

The emergency medical service system (EMSS) is a public health system for quick recognition and transport of emergent patients from the scene to a medical facility. EMSS is usually categorized into three domains, such as prehospital (at scene), transport, and hospital stages. To improve the outcome and prognosis of the transported patients, the three stages of EMSS should be well organized and connected. ${ }^{1}$ For infants and children, the critical role of the 'transport' stage of EMSS has been emphasized, because the most significant causes of mortality among pediatric patients are known to be injury and intoxication., ${ }^{2,3}$

In the Unites States, significant improvements in EMSS was achieved in the 1970s, especially for trauma and cardiac arrest victims. However, the endeavor was mainly focused on adults, and some studies revealed that the improvement in prognosis among pediatric patients was suboptimal in spite of the development of a modern EMSS. ${ }^{4}$ For this reason there was strong suggestion that a pediatric EMSS should be established separately from that of adults, and consequently, an emergency medical service for children was introduced to take care of and transport pediatric patients properly in $1985 .{ }^{5}$ In Korea, EMSS mainly for adult has been developed rapidly through the 1990's and achieved many meaningful goals, such as improved outcomes of cardiac arrest patients, however there is still a significant lack of appropriate investment in infrastructure of pediatric EMSS.

Understanding the realistic features of EMSS is essential to achieve improvements in the EMSS of the country. These data can be used to devlop policies for EMSS and to establish proper education, research and public relations programs. In the United States, each state has multiple EMSS providers which work for the population of the designated area. This system can be a barrier to understanding the comprehensive state of EMSS in each region, and the population-based studies can be hard to conduct. For this probable reason, the epidemiologic studies on pediatric EMSS in the United States are rare. ${ }^{4}$

On the other hand, Korea has a single, centralized EMSS run by the National Emergency Management Agency (NEMA) with the call code 119, as equivalent to 911 in the United States. The 119 crew, mainly emergency medical technicians and registered nurses, has duty to record a 'run sheet' which contains brief information on the transported patients. The data from the run sheets are converted into an electronic database and stored in the information system of NEMA.

The aim of this study was to elucidate the epidemiology of pediatric patients transported in Korea through the EMSS, by analysis of the run sheet log recorded by the 119 rescue service crew.

\section{METHODS}

We extracted epidemiologic data from the collected run sheets, such as age and gender of the pediatric patients, the time, day, season, and year of the requested calls, consciousness level of the patients (alert, responds to verbal stimuli, responds to painful stimuli, or unresponsive; AVPU), main reason for the requested call (disease or injury), and emergency care provided by the 119 crew during the transportation.

We divided the cases into four groups according to the age of the patients, 0 to 1,2 to 5,6 to 10 , and 11 to 15 years old. The emergency management provided by the NEMA crew for arrest victims was also collected such as bag-valve-mask ventilation, monitoring electrocardiography, and intravenous access. To investigate the regional differences in the country, we analyzed the data from 7 metropolitan cities and 9 provinces.

We compared average annual transport numbers of 16 metropolitan cities and provinces to understand the differences in prehospital transport characteristics of each region. We also obtained the adjusted incidence rate (per 100,000 children) of transportation of pediatric patients in each region, using the data provided by Statistics Korea. This study was approved by the institutional review board of Seoul Medical Center (2013-015).

\section{Statistical analysis}

Continuous data were represented by median and interquartile range. Categorical values were reported with frequency (\%). The comparison of demographics of the transport by age group, time, month, and season was analyzed with chi square tests. A P-value less than 0.05 was regarded as statistically significant. Statistics package STATA ver. 14.0 (StataCorp., College Station, TX, USA) was used for statistical analysis.

There were small portions of missing data in the variables of gender, AVPU, and run time. To avoid introducing bias or affecting the representativeness of our result, we performed imputation method for missing values. We used a univariate imputation strategy for each missing value, predictive mean matching for continuous variables, logistic regression for binary variables, and multinomial logistic regression for nominal variables. We used the multiple imputation command in STATA ver. 14.0.

\section{RESULTS}

\section{Subjects}

From January 2006 to December 2008 (3 years), there were a total of 3,672,932 run sheets which were electronically submitted to NEMA by 119 crews in Korea. Among these records, we ob- 
Table 1. Demographics of pediatric ( $<16$ years old) transports by National Emergency Management Agency in Korea

\begin{tabular}{|c|c|c|c|c|c|}
\hline & & Disease & Injury & P-value & Total \\
\hline Total & & 100,893 (100.0) & 137,751 (100.0) & & $238,644(100.0)$ \\
\hline \multirow[t]{4}{*}{ Year } & & & & $<0.001$ & \\
\hline & 2006 & $30,884(30.6)$ & $40,403(29.3)$ & & 71,287 (29.9) \\
\hline & 2007 & 33,466 (33.2) & $47,141(34.2)$ & & 80,607 (33.8) \\
\hline & 2008 & $36,543(36.2)$ & $50,207(36.4)$ & & $86,750(36.4)$ \\
\hline \multirow[t]{3}{*}{ Sex } & & & & $<0.001$ & \\
\hline & Male & $56,303(55.8)$ & $84,594(61.4)$ & & $140,897(59.0)$ \\
\hline & Female & $44,590(44.2)$ & $53,157(38.6)$ & & 97,747 (41.0) \\
\hline \multirow[t]{5}{*}{ Age (yr) } & & & & $<0.001$ & \\
\hline & $0-1$ & $20,211(20.0)$ & 18,734 (13.6) & & 38,945 (16.3) \\
\hline & $2-5$ & $40,440(40.1)$ & $33,487(24.3)$ & & $73,927(31.0)$ \\
\hline & $6-10$ & $19,280(19.1)$ & 39,767 (28.9) & & $59,047(24.7)$ \\
\hline & $11-15$ & 20,962 (20.8) & 45,763 (33.2) & & 66,725 (28.0) \\
\hline \multirow[t]{5}{*}{ Time of day } & & & & $<0.001$ & \\
\hline & $0: 00-5: 59$ & 20,545 (20.4) & $8,244(6.0)$ & & $28,789(12.1)$ \\
\hline & $6: 00-11: 59$ & $21,884(21.7)$ & 22,861 (16.6) & & 44,745 (18.7) \\
\hline & $12: 00-17: 59$ & $26,587(26.4)$ & $60,106(43.6)$ & & $86,693(36.3)$ \\
\hline & 18:00-23:59 & $31,877(31.6)$ & $46,540(33.8)$ & & 78,417 (32.9) \\
\hline \multirow[t]{8}{*}{ Day of week } & & & & $<0.001$ & \\
\hline & Sunday & $14,638(14.5)$ & $22,842(16.6)$ & & $37,480(15.7)$ \\
\hline & Monday & $15,104(15.0)$ & $17,780(12.9)$ & & 32,884 (13.8) \\
\hline & Tuesday & 13,926 (13.8) & $17,800(13.0)$ & & 31,806 (13.3) \\
\hline & Wednesday & $13,759(13.6)$ & $17,960(13.0)$ & & $31,719(13.3)$ \\
\hline & Thursday & $14,383(14.3)$ & $18,025(13.1)$ & & $32,408(13.6)$ \\
\hline & Friday & $14,799(14.7)$ & 19,585 (14.2) & & 34,384 (14.4) \\
\hline & Saturday & $14,284(14.2)$ & 23,679 (17.2) & & 37,963 (15.9) \\
\hline \multirow[t]{5}{*}{ Season of year } & & & & $<0.001$ & \\
\hline & Spring (March to May) & $27,858(27.6)$ & $35,844(26.0)$ & & $63,702(26.7)$ \\
\hline & Summer (June to August) & $24,660(24.4)$ & $40,455(29.4)$ & & $65,115(27.3)$ \\
\hline & Fall (September to November) & $21,384(21.2)$ & $34,877(25.3)$ & & $56,261(23.6)$ \\
\hline & Winter (December to February) & 26,991 (26.8) & $26,575(19.3)$ & & $53,566(22.4)$ \\
\hline \multirow[t]{13}{*}{ Place of call } & & & & $<0.001$ & \\
\hline & Home & $76,060(75.4)$ & $53,568(38.9)$ & & $129,628(54.3)$ \\
\hline & Road & $2,682(2.7)$ & $38,205(27.7)$ & & 40,887 (17.1) \\
\hline & Public facilities & $3,436(3.4)$ & $9,617(7.0)$ & & $13,053(5.5)$ \\
\hline & School & $3,013(3.0)$ & $7,167(5.2)$ & & $10,180(4.3)$ \\
\hline & Hospital & $2,992(3.0)$ & $1,420(1.0)$ & & 4,412 (1.8) \\
\hline & Industrial facilities & $1,282(1.3)$ & $2,007(1.5)$ & & $3,289(1.4)$ \\
\hline & Lodging facilities & $1,046(1.0)$ & $1,433(1.0)$ & & $2,479(1.0)$ \\
\hline & Outdoors & $240(0.2)$ & $1,776(1.3)$ & & $2,016(0.8)$ \\
\hline & Commercial facilities & $423(0.4)$ & $494(0.4)$ & & $917(0.4)$ \\
\hline & Subway & $83(0.1)$ & $122(0.1)$ & & $205(0.1)$ \\
\hline & Others & $7,124(7.1)$ & $16,847(12.2)$ & & $23,971(10.0)$ \\
\hline & Unknown & $2,512(2.5)$ & $5,095(3.7)$ & & $7,607(3.2)$ \\
\hline \multirow[t]{3}{*}{ Region } & & & & $<0.001$ & \\
\hline & Metropolitan level & $47,241(46.8)$ & $54,751(29.7)$ & & 101,992 (42.7) \\
\hline & Province level & $53,652(53.2)$ & $83,000(60.3)$ & & 136,652 (57.3) \\
\hline \multicolumn{6}{|l|}{ AVPU score } \\
\hline & Alert & 80,715 (33.8) & $127,781(53.5)$ & $<0.001$ & $208,496(87.4)$ \\
\hline & Verbal & $12,043(5.0)$ & $5,648(2.4)$ & & $17,691(7.4)$ \\
\hline & Painful & 6,615 (2.8) & $2,787(1.2)$ & & $9,402(3.9)$ \\
\hline & Unresponsive & $1,520(0.6)$ & 1,535 (0.6) & & $3,055(1.3)$ \\
\hline
\end{tabular}

Values are presented as number (\%).

$A$, alert; $V$, response to verbal stimuli; $P$, response to painful stimuli; $U$, unresponsive. 
tained 238,644 (6.5\%) records for pediatric patients (under 16 years old), after eliminating cases of adults, and the cases with uncertain age $(14,551 ; 0.4 \%)$. The mean numbers of pediatric cases per year was 79,548 . There were some cases with missing data on gender $(4,302 ; 1.8 \%)$, consciousness level presented by AVPU method $(4,576 ; 1.9 \%)$, and the run time from the call to scene or scene to hospital $(3,785 ; 1.6 \%)$.

\section{Demographic characteristics of the transported pediatric patients}

The annual average number of transported pediatric patients by the NEMA crew during study period was 79,548 (standard devia- tion $[S D], \pm 6,357$ ). The median age of children was 6.0 (interquartile range, 2 to 11), and boys represented more than half ( $n=140,897 ; 59.0 \%$ ). The most common age among the children was 2 years old $(n=23,190 ; 9.7 \%)$. The most commonly transported age group was the 2- to 5-year-old group (31.0\%). The NEMA services were most frequently provided to the pediatric patients in the afternoon (12:00 p.m. to 17:59 p.m., 36.3\%), on Saturday and Sunday (15.9\% and 15.7\%), and in summer (June through August, 27.3\%). The most common requested site was the home (54.3\%) of the victims (Table 1).

Table 2. Clinical characteristics of pediatric patients transported by National Emergency Management Agency and emergency medical service performance by mental status

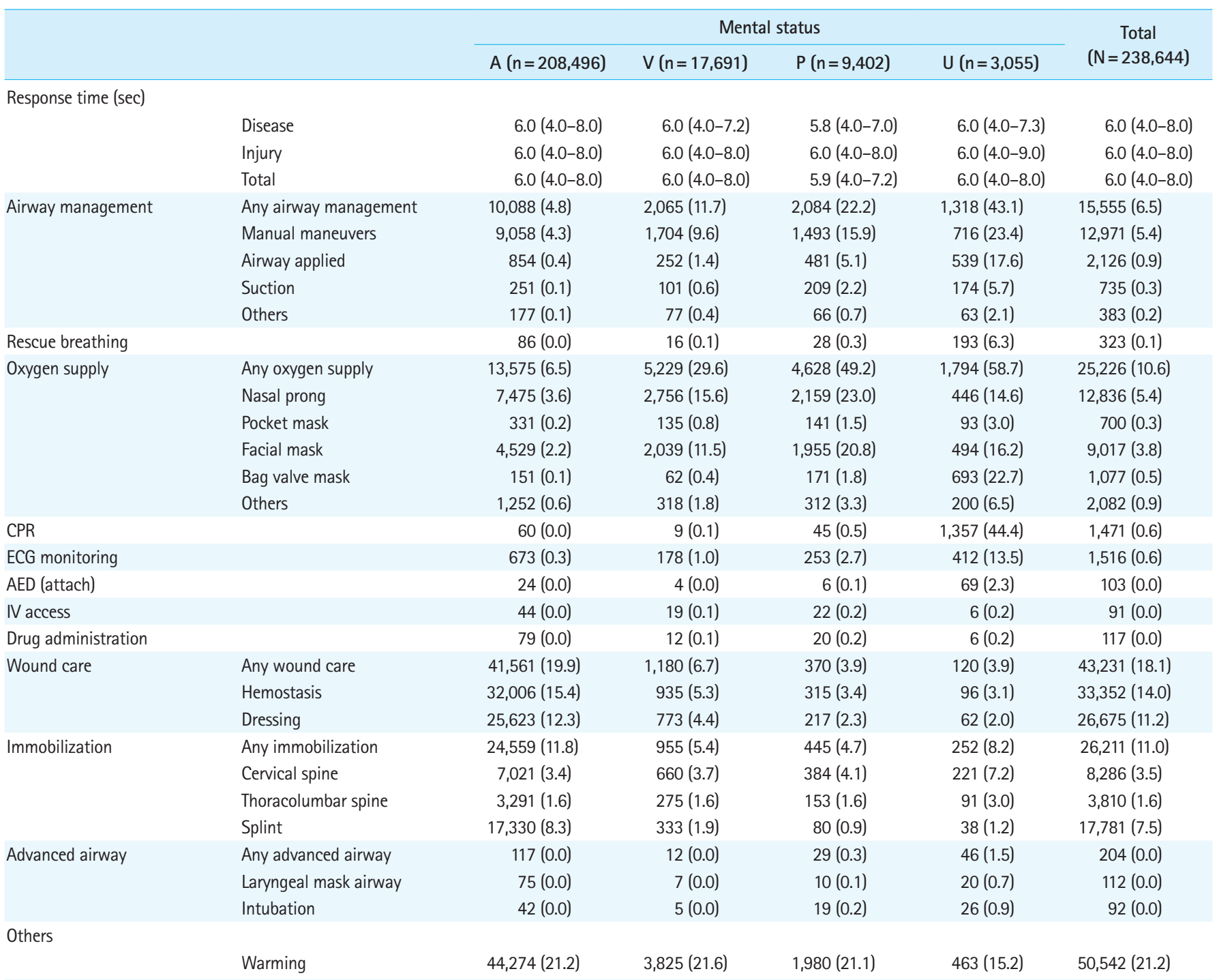

Values are presented as median (interquartile range) or number (\%). Some children received more than one maneuvers in one category. Missing values were excluded from the table and included as denominator.

$A$, alert; $V$, response to verbal stimuli; $P$, response to painful stimuli; $U$, unresponsive; $C P R$, cardiopulmonary resuscitation; ECG, electrocardiography; $A E D$, automated electrical device; IV, intravenous. 


\section{Clinical characteristics of the transported pediatric pa- tients}

When we divided the chief complaints of the patients into two major groups, disease and injury, there were more patients in the injury $(n=137,751 ; 57.7 \%)$ than the disease group $(n=100,893$; $42.3 \%)$. In the disease group, there were more boys (55.8\%) and the largest age group was 2 to 5 years olds (40.1\%). The children in the disease group were most commonly transported in the evening (18:00 p.m. to 23:59 p.m., 31.6\%), on Monday and Friday (15.0\% and 14.7\%, respectively), in spring (March through May, 27.6\%), and from the home of the children (75.4\%). In the injury group, there were more boys (61.4\%) and the largest age group was older than the disease group ( 11 to 15 years old, 33.2\%). The injured children were most frequently moved in the afternoon (12:00 p.m. to 17:59 p.m., 43.6\%), on Saturday and Sunday (17.2\% and $16.6 \%$, respectively), and in summer (June through August, 29.4\%). Most of the traumatized pediatric victims were transported from home (38.9\%) and from the road (27.7\%) (Table 1).

The relative proportions of the consciousness levels of the children judged with the AVPU method by the NEMA crew was 'alert' $(n=208,496 ; 87.4 \%)$, 'responsive to verbal stimuli' $(n=17,671$; $7.4 \%)$, 'responsive to painful stimuli' $(n=9,402 ; 3.9 \%)$, and 'unresponsive' $(n=3,055 ; 1.3 \%)$ (Table 1).

\section{Emergency management provided by NEMA crew}

A total of 186,702 emergency maneuvers were provided to 129,006 children ( $54.1 \%$ of total transported children) by the NEMA crew during the study period (average, 1.45 maneuvers per 1 transport). The emergency interventions were mainly 'heat preservation' (21.2\%), 'wound care' (18.1\%), 'immobilization' (11.0\%), 'oxygen supply' (10.6\%), and 'airway protection' (6.5\%) (Table 2).

The NEMA crew performed a total of 1,471 cardiopulmonary resuscitations. The proportions of the resuscitative procedures provided during cardiopulmonary resuscitation were as follows: 'bag-valve-mask ventilation' ( $n=556,37.8 \%)$, 'monitoring of electrocardiography' $(n=268,18.2 \%)$ and 'vascular access' $(n=3$, $0.2 \%)$.

\section{Comparison of the pediatric prehospital transports among metropolitan cities and provinces in Korea}

We analyzed and compared the prehospital transport services across 7 metropolitan cities (Seoul, Busan, Incheon, Daegu, Daejeon, Gwangju, and Ulsan) and 9 provinces (Gyeonggi, Gangwon, Chungbuk, Chungnam, Gyeongbuk, Gyeongnam, Jeonbuk, Jeonnam, and Jeju) in Korea. The mean absolute number of the pediatric transports was highest in Gyeonggi $(n=20,467.7 ; 26.7 \%$; SD, $\pm 2,523.8)$,
Seoul $(n=14,030.7 ; 18.3 \%$; SD, \pm 563.2$)$, and Incheon $(n=5,587.7$; $7.3 \% ; \mathrm{SD}, \pm 1,476.5)$ in decreasing order. The sum of transports in these three regions was about half of the total transported patients (50.0\%). The smallest numbers were transported annually in Chungbuk $(n=2,350.0 ; 3.1 \%$; $S D, \pm 361.5)$, Jeju $(n=1,803.7 ; 2.3 \%$; $S D$, $\pm 47.7)$, and Ulsan ( $n=1,687.7 ; 2.2 \% ; S D, \pm 130.7)$.

We converted these absolute numbers to the incidence of the transported cases per 100,000 pediatric population numbers standardized by age, and gender to the Korean child population. The national average transport per 100,000 children was 992.6. When we compared this incidence per 100,000 children among the 16 regions in Korea, Jeju was the highest $(n=1,650.2 ; 10.4 \%)$, followed by Gangwon $(n=1,201.3 ; 7.6 \%)$, Jeonnam $(n=1,178.1$; $7.4 \%)$ and Incheon $(n=1,172.5 ; 7.4 \%)$. The lowest incidence per 100,000 pediatric population was observed in Busan $(n=770.6$, 4.9\%), Gyeongnam ( $n=758.5,4.8 \%)$, and Daegu $(n=601.6,3.8 \%)$ (Table 3 and Fig. 1).

\section{DISCUSSION}

In this study, we describe the nation-wide characteristics of pediatric prehospital transports over 3 years in Korea. Although previous small studies showed the regional features of general or pediatric transports provided by NEMA, those studies have been limited by the small numbers of the subjects in any single region. ${ }^{6}$ The most important strength of our study is that this is the first national report on the pediatric prehospital transport in Korea.

Table 3. Standardized annual incidence of pediatric transports by $\mathrm{Na}$ tional Emergency Management Agency crews in Korea ${ }^{\text {a) }}$

\begin{tabular}{lrrrr}
\hline & 2006 & 2007 & 2008 & Average \\
\hline Seoul & 906.6 & 951.9 & 983.6 & 947.4 \\
Busan & 703.1 & 781.6 & 827.2 & 770.6 \\
Daegu & 371.8 & 680.6 & 752.4 & 601.6 \\
Incheon & 858.7 & $1,181.4$ & $1,477.3$ & $1,172.5$ \\
Gwangju & $1,213.2$ & $1,003.2$ & $1,097.9$ & $1,104.8$ \\
Daejeon & $1,085.7$ & $1,181.9$ & $1,100.2$ & $1,122.6$ \\
Ulsan & 733.3 & 806.1 & 855.1 & 798.2 \\
Gyeonggi & 806.3 & 928.3 & $1,033.2$ & 922.6 \\
Gangwon & $1,096.4$ & $1,238.4$ & $1,269.2$ & $1,201.3$ \\
Chungbuk & 741.7 & 878.6 & $1,012.1$ & 877.5 \\
Chungnam & 997.7 & $1,050.8$ & $1,014.3$ & $1,020.9$ \\
Jeonbuk & 856.6 & 898.3 & 962.4 & 905.8 \\
Jeonnam & 875.1 & 1210.4 & 1448.8 & $1,178.1$ \\
Gyeongbuk & 797.4 & 874.4 & 874.5 & 848.8 \\
Gyeongnam & 697.3 & 782.5 & 795.7 & 758.5 \\
Jeju & $1,599.0$ & $1,667.9$ & $1,683.7$ & $1,650.2$ \\
\hline
\end{tabular}

a)Incidence rates are standardized by age and gender to the Korean child population. 

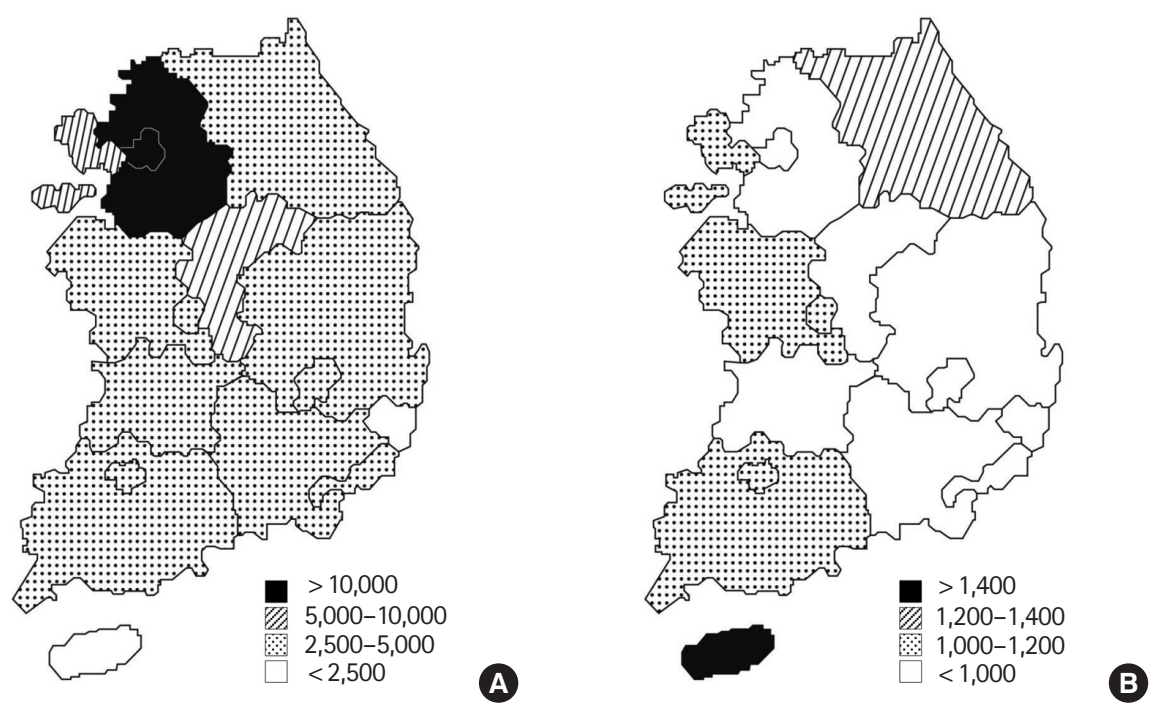

Fig. 1. The number of pediatric prehospital transports by National Emergency Management Agency per 100,000 children in Korea. (A) Average number of pediatric transports per year. (B) Average number of pediatric transports per 100,000 children per year. Incidence rates are standardized by age and gender to the Korean child population.

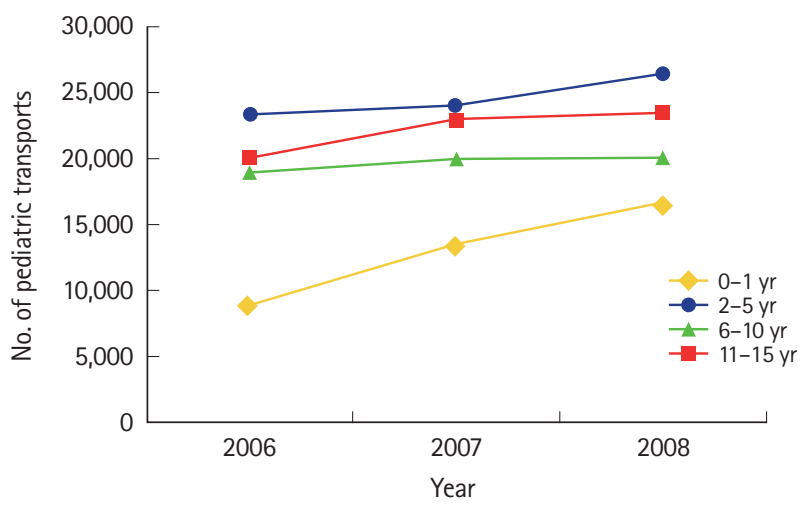

Fig. 2. Temporal trend of number of pediatric prehospital transports by National Emergency Management Agency by year and age group.

In the United States, the proportion of pediatric transports out of the total prehospital transports is known to be $4 \%$ to $13 \%$, and only $5 \%$ to $7 \%$ of the children are brought to emergency departments using the EMSS..$^{72}$ The children transported by EMSS had about a three times higher admission rate than the children brought by other vehicles. ${ }^{11,12}$ During the three years of the study, the pediatric proportion of the prehospital transports was 6.5\% $(n=238,644)$. Over time, the proportion slowly but steadily increased, from 6.3\% ( $n=71,287$; in 2006), 6.5\% $(n=80,607$; in 2007), and 6.6\% ( $n=86,750$; in 2008) (Fig. 2). A recently published study reported that the pediatric proportion of the EMSS in 124 Korean emergency departments was $4.2 \%$, and that the admission rate was $14.1 \%$, which is 2.5 times higher than in the group of patients who were transported otherwise. ${ }^{13}$
Considering these data, there is significant need for advanced prehospital transport in Korea because there are significant and increasing numbers of the severe pediatric cases who require effective EMSS.

A cross-sectional study of pediatric (less than 19 years old) prehospital transports in 112 emergency departments of the united State showed that the most common pediatric age group was 12 to 18 years old (46\%), and that girls were 52\%. According to this study, injured children represented more than half (54\%), and the most frequently transported time and season were 16:00 p.m. to 23:59 p.m. (47.0\%), and in summer (June to August, $31 \%$ ). ${ }^{12}$ Although our study used a different age definition for the pediatric patients (less than 16 years old) than that of the American study, the most common Korean children age group was relatively younger (2 to 5 years old, $31.0 \%$ ), and prehospital transports were most frequently requested at a relatively earlier time (12:00 p.m. to 17:59 p.m., 36.3\%). This discrepancy suggests that national epidemiologic data is essential to establish and to develop specific prehospital transport systems in each country.

This study provides meaningful data which are critical in order to improve the pediatric prehospital care system in Korea. In order to determine the distribution of resources, such as personnel, equipment, and facilities the absolute and population-adjusted pediatric transport numbers of each metropolitan city and province would be helpful. For example, to expand the pediatric transport system, the adjusted pediatric transport cases per 100,000 children of Gangwon and Incheon are higher than those 
of Seoul and Gyeonggi province. Another important implication relates to the most frequently requested time and season. Because the total number of transport calls is largest in the afternoon (12:00 p.m. to 17:59 p.m.), on weekends (Saturday and Sunday), and in the summer, these periods requires more pediatric transport resources. Moreover, the fact that the proportion of pediatric patients with consciousness level 'A (alert)' is significantly lower in the disease group (80.0\%) than the injury group $(92.8 \%)$ indicates that the disease group requires more careful management during the transport.

A total of 1,471 CPRs were performed during the study period. The level of consciousness of most patients was unresponsive (92\%), but there were 60 cases of CPR performed on initially alert children. This could be due to a recording error. However, the clinical course of those patients might deteriorate rapidly during transport. We could not analyze the clinical course of these cases because no detailed clinical data were available in the run sheets.

This study has some limitations. First, there were some missing records in the dataset of the study. This limitation is partly inevitable due to the retrospective nature of the study, and this drawback hampers more detailed analysis of the data. Although percentage of missing records was only 3.5\% (8,521 cases), in some age groups, especially the infant group had more missing records. Second, the quality of run sheets between different regions of the country was variable. For example, the missing rates of the data were $16.3 \%$ and $14.5 \%$ in Incheon and Jeonnam, respectively. This inappropriately high rate of missing data strongly suggests that quality control is warranted in preparation and collection of the run sheets. Third, although we could analyze the prehospital management of the transported children, the clinical outcome of the patients was unclear due to the lack of merged data between prehospital and hospital records. Merging of the databases should be a high-priority for the improvement of the Korean pediatric EMSS.

In summary, we presented comprehensive epidemiologic data of pediatric patients transported by national emergency medical services in Korea, and we hope these data can be used as hard facts to develop and improve the pediatric EMSS in the country.

\section{CONFLICT OF INTEREST}

No potential conflict of interest relevant to this article was reported.

\section{REFERENCES}

1. Brice JH, Valenzuela $T$, Ornato JP, et al. Optimal prehospital cardiovascular care. Prehosp Emerg Care 2001;5:65-72.

2. United Nations Children's Fund. A league table of child deaths by injury in rich countries [Internet]. Florence: United Nations Children's Fund Innocenti Research Centre; 2001 [cited 2013 Dec 30]. Available from: http://www.unicef-irc.org/publications/pdf/repcard2e.pdf.

3. Statistics Korea. Annual report on the cause of death statistics 2009 [Internet]. Daejeon: Statistics Korea [cited 2017 Jun 1]. Available from: http://kostat.go.kr/portal/korea/kor_nw/2/6/2/ index.board.

4. Shah MN. The formation of the emergency medical services system. Am J Public Health 2006;96:414-23.

5. Krug S, Kuppermann N. Twenty years of emergency medical services for children: a cause for celebration and a call for action. Pediatr Emerg Care 2005;21:223-6.

6. Jeong JS, Hong KJ, Shin SD, Suh GJ, Song KJ. Evaluation of the appropriateness of prehospital emergency care by 119 rescue services in seoul metropolitan area. J Korean Soc Emerg Med 2008;19:233-44.

7. Kost $\mathrm{S}$, Arruda J. Appropriateness of ambulance transportation to a suburban pediatric emergency department. Prehosp Emerg Care 1999;3:187-90.

8. Seidel JS, Hornbein M, Yoshiyama K, Kuznets D, Finklestein JZ, St Geme JW Jr. Emergency medical services and the pediatric patient: are the needs being met? Pediatrics 1984;73:769-72.

9. Foltin GL, Pon S, Tunik $M$, et al. Pediatric ambulance utilization in a large American city: a systems analysis approach. Pediatr Emerg Care 1998;14:254-8.

10. Suruda A, Vernon DD, Reading J, et al. Pre-hospital emergency medical services: a population based study of pediatric utilization. Inj Prev 1999;5:294-7.

11. Johnston C, King WD. Pediatric prehospital care in a southern regional emergency medical service system. South Med J 1988;81:1473-6.

12. Shah MN, Cushman JT, Davis CO, Bazarian JJ, Auinger $P$, Friedman B. The epidemiology of emergency medical services use by children: an analysis of the National Hospital Ambulatory Medical Care Survey. Prehosp Emerg Care 2008;12:26976.

13. Kwak YH, Kim DK, Jang HY. Utilization of emergency department by children in Korea. J Korean Med Sci 2012;27:1222-8. 